\title{
General Practitioners use of the Cochrane Library in London
}

\author{
Felix S F Ram, Sheree R Wellington
}

\author{
Abstract \\ Aim \\ To investigate the use of the Cochrane Library by general practitioners (GPs) \\ in London.

\begin{abstract}
Method
We conducted a postal questionnaire survey of general practices in South London. Two hundred and eighty-seven questionnaires were posted in December 2001 to all general practices in the South London region. All addresses beginning with either a SE or SW postcode were sent a two-page
\end{abstract} \\ questionnaire with a covering letter.
}

\section{Results}

The response rate was $30 \%$, with $33 \%$ of GPs having ever used the Cochrane Library and $67 \%$ that had not previously used the library. General practitioners that reported to have used the Cochrane Library used it only occasionally and found the library to be user friendly as well as being a useful source of information. However, 52\% of GPs had never heard or used the Cochrane Library, and most reported poor publicity, awareness and time constrains as the major reasons for their lack of use.

\section{Conclusions}

The Cochrane Library of systematic reviews is a considered a user-friendly and useful source of evidence-base information. However, the utilisation and uptake of the Cochrane Library by GPs in South London is poor. Further resources are required to improve utilisation and increase awareness of this important source of evidence in primary care.

\section{Dr Felix S F Ram \\ Research Fellow in Respiratory Medicine}

\section{Sheree R Wellington}

Clinical Pharmacist

Correspondence to:

Dr Felix S F Ram

Cochrane Airways Group

St. Georges Hospital

Medical School

Department of

Physiological Medicine

Level 0, Jenner Wing

Cranmer Terrace, Tooting

London, SW17 0RE

Email: fram@sghms.ac.uk

Date submitted: $27 / 05 / 02$

Date Accepted: 08/11/02

Prim Care Resp J 2002:

11(4);123-124

Figure 1: User friendliness of the Cochrane Library as reported by general practitioners in South London.

Do you think the Cochrane Library is user friendly?
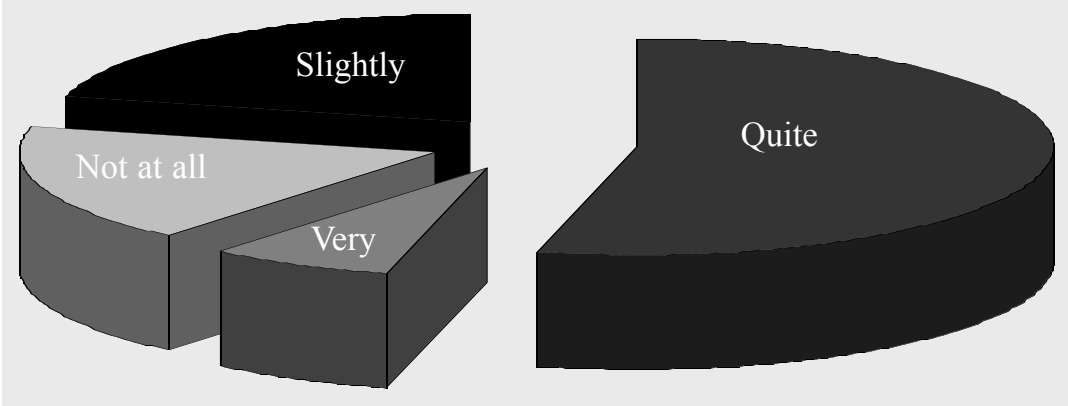

systematic reviews and reviews of effectiveness) is being utilised. It is important to know if the medical evidence collected and reviewed by the Cochrane Collaboration is utilised by primary care doctors, who are the health professionals most often seen by members of the general public.

\section{Objectives}

To estimate the use of the Cochrane Library among GPs in South London by conducting a postal questionnaire survey.

\section{Methods}

A two-page questionnaire with 11 questions was sent out with a cover letter to 287 general practices in the South London region. The general practice postal addresses were obtained from the Local NHS website link of the Department of Health website (http://www.doh.gov.uk/). All addresses beginning with either a SE or SW postcode were abstracted from this NHS website and entered into a database for the purpose of the questionnaire.

Results

At the end of March 2002 the response rate was 30\% (86) from the 287 posted questionnaires. Thirty-three percent (28) of GPs had used the Cochrane Library previously and $67 \%$ (58) had never used the Cochrane Library.

Of the GPs that had used the Cochrane Library previously, $85 \%$ had used the Cochrane Library seldom or occasionally. Fifty-four percent of GPs found the information on the Cochrane Library quite user friendly, $7 \%$ found it very user friendly, $21 \%$ found it only slightly user friendly while $18 \%$ did not find it user friendly at all (Figure 1). Fifty-three percent of GPs found the information contained on the Cochrane Library quiet useful, $36 \%$ found it very useful and $11 \%$ found the information only slightly or not at all useful. Most GPs (44\%) had used the Cochrane Library for patient management, $43 \%$ for study or research purposes and $10 \%$ used it to help prepare for presentations or seminars. The majority of GPs (75\%) had used the Cochrane Library for either the database of completed systematic 
reviews or the reviews of effectiveness. Only $15 \%$ of GPs had used the Cochrane trials register on the Cochrane Library. The most useful sections of the library were reported by $87 \%$ of GPs to be the database of reviews and reviews of effectiveness. The most frequently used section of a Cochrane review on the library was the abstract ( $43 \%$ ) followed by the use of the whole review (26\%), synopsis $(20 \%)$ and conclusions $(11 \%)$. Most GPs $(82 \%)$ rated the standard of the reviews on the Cochrane Library as either good or excellent, with $14 \%$ rating the Cochrane Library as adequate and $4 \%$ as poor (Figure 2).

Of the 58 GPs that had not used the library before $52 \%$ had never heard of the Cochrane Library previously. Thirty-one percent of GPs did not utilise the Cochrane Library due to time constrains, $9 \%$ were aware of the Cochrane Library but did not understand its usefulness, $5 \%$ had claimed to have no use for the Cochrane Library and 3\% of GPs provided other explanations for not using the Cochrane Library. These included search strategy on the Cochrane Library being too difficult; practice used alternative sources of evidence and used only print journals. GPs that responded no previous use of the Cochrane Library were given the opportunity to comment on how their use of the Cochrane Library could be improved. Eleven percent wanted better access facilities, $24 \%$ requested further information on the Cochrane Library and $25 \%$ suggested seminars on the use of the Cochrane Library in order to increase publicity and awareness.

\section{Discussion}

The Cochrane Library of systematic reviews is considered a user-friendly and useful source of evidencebase information for only a handful of GPs in South London. The majority of GPs that have previously used the Cochrane Library found the standard of the reviews on the library of good quality. However, the use and uptake of the Cochrane Library by GPs in South London is poor. A survey published in $1998^{1}$ that was conducted in the former Wessex region (South England) showed that the rate of use of the Cochrane Library by GPs was 4\% (11/284 respondents). A year later, an Australian survey ${ }^{2}$ reported the rate of use among GPs to be $10 \%$ (13/134 respondents). It is encouraging to learn from the current survey that the rate of use of the Cochrane Library in London by GPs is 33\% (28/86 respondents). This higher figure compared to the Wessex region survey could be due to the greater availability of the Cochrane Library within the UK health sector over the years.

The response rate of $30 \%$ for our postal questionnaire was small this is not surprising as response rates to questionnaire surveys among GPs are dropping. ${ }^{3}$ However, it is likely that the GPs that returned the questionnaire would have actually heard of the existence of the Cochrane Library, whether they had used it or not. Therefore, it is possible that we have overestimated the rate of use of the Cochrane Library in general practice.

The main reason for not using the Cochrane Library was lack of awareness or publicity. Most of the GPs requested further information in way of seminars to be held in their area and or written information to be sent by post or
Figure 2: Standard of systematic reviews on the Cochrane Library as rated by general practitioners in South London.

\section{How would you rate the standard of reviews on the Cochrane Library}

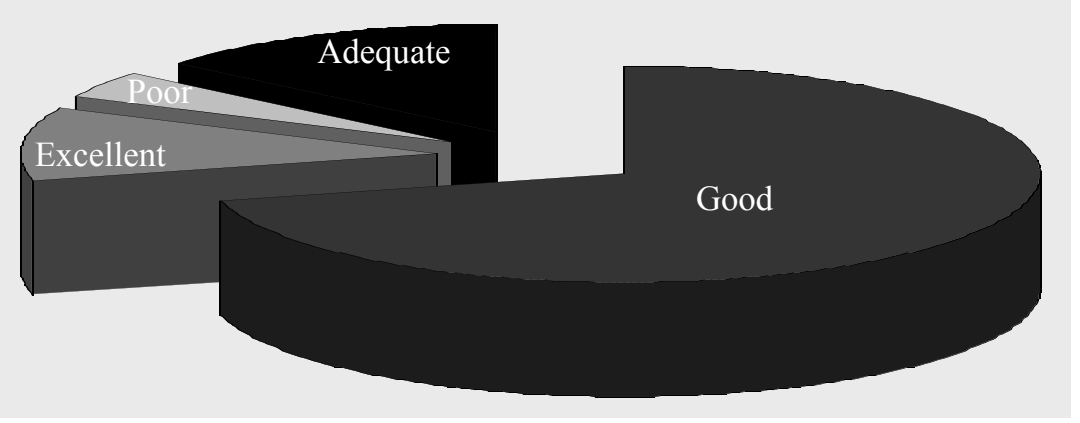

email. Another important reason for not having ever used the library was time constraints. This finding suggests that in order to increase utilisation of the Cochrane Library and or other resources of evidence based medicine in general practice, information should be available in the clinic within a short time. Such information could be provided by an independent source much like support for biochemistry or pathology services.

\section{Conclusions}

Large amounts of the UK NHS R\&D funding support the work of the Cochrane Collaboration in the UK.

Therefore, although there has been an encouraging increase in the rate of use of the Cochrane Library it is important to further increase the utilisation of the work of the Collaboration by GPs, not only in South London but also throughout the UK. In this age of "evidence-based medicine" it is important that GPs are aware of such an important source of evidence, which is freely available to all persons with access to the internet in the UK (http://www.nelh.nhs.uk/cochrane.asp). Further resources are required to improve utilisation and increase awareness of this important source of evidence among primary care physicians in the UK.

\section{Acknowledgement}

Dr Felix Ram is supported by a fellowship grant from the Netherlands Asthma Foundation, The Netherlands.

\section{References:}

1. McColl A, Smith H, White P, Field J. General practitioner's perceptions of the route to evidence based medicine: a questionnaire survey. BMJ 1998;316:361-5. 2. Young JM, Ward JE. General practitioners' use of evidence databases. Med J Aust 1999;170:56-8. 3. McAvoy BR, Kaner EF. General practice postal surveys: a questionnaire too far? BMJ 1996;313:732-3. 